Jurica Sorić

Tomislav Lesičar

Filip Putar

Zdenko Tonković

http://dx.doi.org/10.21278/brod72407

ISSN 0007-215X

eISSN $1845-5859$

\title{
ON MULTISCALE DAMAGE MODELLING OF HETEROGENEOUS MATERIALS USING NONLOCAL CONTINUUM THEORY
}

UDC 620.178.2

Review paper

\begin{abstract}
Summary
An overview of the modelling of quasi-brittle as well as ductile damage is given. The multiscale procedure employing the nonlocal continuum theory is described in more detail. The softening is introduced at the microlevel in the microstructural volume element and after that the homogenization procedure state variables are mapped at the macrolevel material point via the scale transition approach. In the case of quasi-brittle softening the $C^{1}$ continuous finite element discretization is applied at both micro- and macrolevel. At the modelling of ductile damage response, the macrolevel is also discretized by the $C^{1}$ finite element formulation, while the microscale utilizes quadrilateral mixed finite elements employing the nonlocal equivalent plastic strain and gradient-enhanced elastoplasticity. All approaches presented are verified in the standard examples.
\end{abstract}

Keywords: $\quad$ heterogeneous material; two-scale approach; computational homogenization; quasi-brittle damage; ductile damage

\section{Introduction}

The modern structures are characterized by rising complexity, where requirements on reliability and efficiency are continuously increasing. To achieve those high requirements, materials with optimized heterogeneous microstructure are exploited, where microstructural topology governs microstructural mechanisms, such as material softening. Furthermore, material microstructure directly influences aging mechanisms, for example, corrosion, and fatigue, which consequently may lead to a structural collapse. Therefore, in order to assess structural integrity and to predict structural lifetime, an analysis dealing with microstructure is necessary. Derivation of an efficient multiscale approach is an important challenge in the shipbuilding and related industries [1,2].

The constitutive models assuming material homogeneity are unable to capture the microstructural phenomena. As a remedy, the multiscale framework in combination with homogenization concepts directly considers the essential microstructural phenomena [3-7]. Herein, one of the best suited concepts is the computational homogenization, embedding the 
microstructure into macroscale boundary value problem (BVP) through an additional BVP, representing microscale $[8,9]$. The microstructural $\mathrm{BVP}$ is represented by the Microstructural Volume Element (MVE) and driven by the macroscale strain quantities, which after solving the corresponding BVP feeds the macroscale with material constitutive model $[10,11]$.

Employment of the computational homogenization in a standard manner in modelling of the softening is unfortunately not possible. The issues which arise are questionable MVE representativeness [12, 13], ill-posedness of the macrolevel BVP [14] and violation of the scale separation principle $[15,16]$. An approach which copes with the aforementioned difficulties is the homogenization of interfacial volumes [17]. In continuous-to-discontinuous approaches, material properties in the microscale localization zone are upscaled into the macroscale fracture modelled as a weak discontinuity [18] or a strong discontinuity [19, 20]. As a possibility, the coupled volume method [14] can be also used. An extension of the methodology proposed in [20] is derived in [21, 22], relying again on the averaging throughout failure zone. In [23, 24] a consistent method incorporating the modified boundary conditions is proposed, resolving the quasi-brittle damage in heterogeneous materials.

For multiscale modelling of ductile damage, in [25] a strong coupling methodology with implicit integration is derived. In [26], a 3D multiscale methodology for damage modelling in polycrystalline materials is proposed, where objectivity is ensured through the application of nonlocal strain terms. Furthermore, in [27], ductile softening of hyperelastic materials adopting large strain assumption is investigated by means of the mean field homogenization and the multiplicative decomposition of deformation gradient. In [28], the mean field approach is extended by utilization of the incremental micromechanics scheme along with isotropization, and it is applied for modelling of damage in composites. In [29], the model parameters of Gurson-Tvergaard-Needleman (GTN) porous plasticity have been calibrated by the homogenization on the MVE discretized by an enriched Voronoi cell. A methodology mentioned in [23] is extended to the consideration of ductile damage in [30]. Furthermore, the full-field homogenization has been performed on the MVE obtained from the microscopic images as presented in [31]. A detailed overview of the state of the art is given in [32-34]. In spite of many possible solutions, neither of them offers an efficient way for consistent multiscale modelling of heterogeneous materials during softening.

The authors have proposed a first-order micro-macro scheme dealing with ductile softening in [35]. The homogenization is conducted on the two MVEs, the first without damage, considering elastoplastic behaviour, and the second with the ductile damage included. To obtain the macroscale constitutive operator, the additional microstructural BVPs are solved providing the relation of damage variable and macrostrain components. The mesh objectivity at the MVE exhibiting formation of the localization zone is ensured by the discretization with mixed finite element enriched by the nonlocal equivalent plastic strain. Although the derived framework gives physically meaningful results, the homogenization employing multiple MVEs is complex and time consuming.

In this manuscript, a micro-macro algorithm for modelling of quasi-brittle damage is shown, where the macrolevel and microlevel are described by the nonlocal theory. Hence, discretization is performed using the $C^{1}$ continuity finite element already derived in [7] for linear elasticity. Since the nonlocal phenomena are embedded into the numerical model, the difficulties, such as mesh objectivity or spurious damage growth are expected to be alleviated and regularized.

Second part of this paper deals with a conventional second-order micro-macro framework for ductile damage modelling. Herein, the macroscale is described by the nonlocal theory and discretized by the triangular $C^{1}$ finite element. The constitutive behaviour is upscaled from the microlevel in a standard way from a single MVE, compared to [35], where 
five MVEs are applied. Discretization of the microscale is again provided by the quadrilateral mixed finite formulation employing an additional degree of freedom (DOF), i.e., nonlocal equivalent plastic strain. The von Mises gradient-enhanced elastoplasticity is employed for the consideration of plastic yielding and softening. The solutions of the benchmark problems demonstrate physically realistic softening responses.

The paper is organized as follows. After an overview of the state of the art in the numerical modelling of multiscale softening responses given in this Section, the modelling of quasi-brittle damage evolution is presented in Section 2. Therein the finite element formulation employing damage variable is described. Furthermore, the nonlocal multiscale scheme with the basic relations of the macro-micro transition is displayed. The numerical example demonstrating the efficiency is given at the end of the Section. Section 3 is concerned with the ductile damage modelling and it starts with the macrolevel finite element formulation. Thereafter the ductile damage model and the homogenization procedure are presented, and the scale transition scheme is briefly described. The Section ends with the two numerical examples. The paper concluding remarks are given in Section 4.

\section{Modelling of quasi-brittle damage evolution}

\subsection{Finite element formulation for modelling of quasi-brittle damage evolution}

The finite element presented in Fig. 1 has three nodes with 12 DOF in each node. The displacement field within the element is approximated by the condensed fifth order polynomial. The nodal unknowns are two displacements and all their first- and second-order derivatives. The element satisfies $C^{1}$ continuity with the assumption of plane strain.

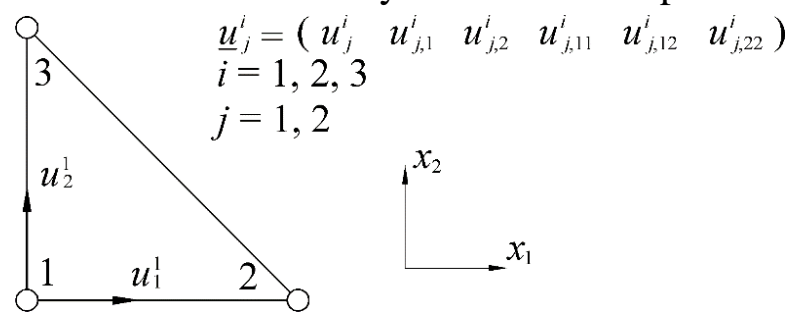

Fig. $1 C^{1}$ continuity triangular finite element

The finite element equation is derived from the virtual work principle which is here expressed in terms of the strain gradients as

$$
\begin{gathered}
\int_{A}(\boldsymbol{\sigma}: \delta \boldsymbol{\varepsilon}) \mathrm{d} A+\int_{A}\left(\boldsymbol{\mu}_{x 1} \vdots \delta \boldsymbol{\varepsilon}_{x 1}\right) \mathrm{d} A+\int_{A}\left(\boldsymbol{\mu}_{x 2}: \delta \boldsymbol{\varepsilon}_{x 2}\right) \mathrm{d} A= \\
\int_{S}(\mathbf{t} \cdot \delta \mathbf{u}) \mathrm{d} s+\int_{s}[\mathbf{T}:(\nabla \otimes \delta \mathbf{u})] \mathrm{d} s .
\end{gathered}
$$

Herein $\boldsymbol{\sigma}$ and $\boldsymbol{\varepsilon}$ are the stress and strain variables, respectively. The higher-order variables $\boldsymbol{\varepsilon}_{x 1}$ and $\boldsymbol{\varepsilon}_{x 2}$ are the strain gradients expressed in the following form

$$
\boldsymbol{\varepsilon}_{x 1}=\left[\begin{array}{c}
\varepsilon_{11,1} \\
\varepsilon_{22,1} \\
2 \varepsilon_{12,1}
\end{array}\right]=\mathbf{B}_{\eta 1} \mathbf{v},
$$




$$
\boldsymbol{\varepsilon}_{x 2}=\left[\begin{array}{c}
\varepsilon_{11,2} \\
\varepsilon_{22,2} \\
2 \varepsilon_{12,2}
\end{array}\right]=\mathbf{B}_{\eta 2} \mathbf{v},
$$

where $\mathbf{B}_{\eta 1}$ and $\mathbf{B}_{\eta 2}$ contain the gradients of the strain-displacement matrix $\mathbf{B}_{\varepsilon}$, while $\mathbf{v}$ is the DOF vector. The matrix $\mathbf{B}_{\varepsilon}$ contains appropriate first derivatives of the interpolation polynomials expressed by the matrix $\mathbf{N}$. The displacement field $\mathbf{u}$ is approximated by the standard expression,

$$
\mathbf{u}=\mathbf{N v} \text {. }
$$

The strain tensor $\boldsymbol{\varepsilon}$

$$
\boldsymbol{\varepsilon}=\left[\begin{array}{c}
\varepsilon_{11} \\
\varepsilon_{22} \\
2 \varepsilon_{12}
\end{array}\right]=\mathbf{B}_{\varepsilon} \mathbf{v},
$$

is work conjugated to Cauchy stress tensor

$$
\boldsymbol{\sigma}=\mathbf{C}_{\sigma \varepsilon} \mathbf{B}_{\varepsilon} \mathbf{v}
$$

where $\mathbf{C}_{\sigma \varepsilon}$ is the elasticity matrix. The double stress tensors are written as

$$
\begin{gathered}
\boldsymbol{\mu}_{x 1}=\left[\begin{array}{l}
\mu_{111} \\
\mu_{122} \\
\mu_{112}
\end{array}\right]=l^{2} \mathbf{C}_{\sigma \varepsilon} \mathbf{B}_{\eta 1} \mathbf{v}, \\
\boldsymbol{\mu}_{x 2}=\left[\begin{array}{l}
\mu_{211} \\
\mu_{222} \\
\mu_{212}
\end{array}\right]=l^{2} \mathbf{C}_{\sigma \varepsilon} \mathbf{B}_{\eta 2} \mathbf{v} .
\end{gathered}
$$

In Eq. (1) $s$ and $A$ represent the outside boundary and the surface of the element. $\mathbf{t}$ is the traction and $\mathbf{T}$ is the double traction, which are work conjugated to the displacement and the displacement gradient, respectively. Additionally, the boundary condition $(\nabla \otimes \mathbf{u}) \cdot \mathbf{n}$ should be satisfied to solve the corresponding BVP. As the measure of nonlocality, an additional material parameter representing the size of the microstructure $l^{2}$ is introduced in Eqs. (7) and (8).

Using the discretization performed by Eqs. (2) - (5) and the incrementation procedure according to the consideration of nonlinear problems, as described in [36], the following incremental form of the virtual work principle is derived

$$
\begin{gathered}
\int_{A} \mathbf{B}_{\varepsilon}^{T} \Delta \boldsymbol{\sigma} \mathrm{d} A+\int_{A} \mathbf{B}_{\eta 1}^{T} \Delta \boldsymbol{\mu}_{x 1} \mathrm{~d} A+\int_{A} \mathbf{B}_{\eta 2}^{T} \Delta \boldsymbol{\mu}_{x 2} \mathrm{~d} A= \\
\int_{s}\left(\mathbf{N}^{T} \mathbf{t}+\operatorname{grad} \mathbf{N}^{T} \mathbf{T}\right) \mathrm{d} s-\int_{A}\left(\mathbf{B}_{\varepsilon}^{T} \boldsymbol{\sigma}^{i-1}+\mathbf{B}_{\eta}^{T} \boldsymbol{\mu}^{i-1}\right) \mathrm{d} A .
\end{gathered}
$$

The damage constitutive model can be expressed by the relations

$$
\boldsymbol{\sigma}=(1-D) \mathbf{C}_{\sigma \varepsilon}: \boldsymbol{\varepsilon},
$$




$$
\begin{aligned}
& \boldsymbol{\mu}_{x 1}=l^{2}\left[(1-D) \mathbf{C}_{\sigma \varepsilon}: \boldsymbol{\varepsilon}_{x 1}\right] \\
& \boldsymbol{\mu}_{x 2}=l^{2}\left[(1-D) \mathbf{C}_{\sigma \varepsilon}: \boldsymbol{\varepsilon}_{x 2}\right] .
\end{aligned}
$$

After the standard updating procedure as presented in [36], the constitutive damage model may be written in the incremental form as

$$
\begin{aligned}
& \Delta \boldsymbol{\sigma}=\left(1-D^{i-1}\right) \mathbf{C}_{\sigma \varepsilon} \Delta \boldsymbol{\varepsilon}-\mathbf{C}_{\sigma \varepsilon} \boldsymbol{\varepsilon}^{i-1} \Delta D, \\
& \Delta \boldsymbol{\mu}_{x 1}=l^{2}\left(1-D^{i-1}\right) \mathbf{C}_{\sigma \varepsilon} \Delta \boldsymbol{\varepsilon}_{x 1}-l^{2} \mathbf{C}_{\sigma \varepsilon} \boldsymbol{\varepsilon}_{x 1}^{i-1} \Delta D, \\
& \Delta \boldsymbol{\mu}_{x 2}=l^{2}\left(1-D^{i-1}\right) \mathbf{C}_{\sigma \varepsilon} \Delta \boldsymbol{\varepsilon}_{x 2}-l^{2} \mathbf{C}_{\sigma \varepsilon} \varepsilon_{x 2}^{i-1} \Delta D .
\end{aligned}
$$
equation

Inserting the constitutive relations (13) - (15) into Eq. (9), gives the finite element

$$
\left(\mathbf{K}_{\sigma}+\mathbf{K}_{\mu x 1}+\mathbf{K}_{\mu x 2}\right) \Delta \mathbf{v}=\mathbf{F}_{\mathrm{e}}-\mathbf{F}_{\mathrm{i}}
$$

with the particular element stiffness matrices defined as

$$
\begin{aligned}
& \mathbf{K}_{\sigma}=\int_{A}\left(\mathbf{B}_{\varepsilon}\right)^{T}\left[(1-D)^{i-1} \mathbf{C}_{\sigma \varepsilon} \mathbf{B}_{\varepsilon}-\mathbf{C}_{\sigma \varepsilon} \boldsymbol{\varepsilon}^{i-1}\left(\frac{\mathrm{d} D}{\mathrm{~d} \boldsymbol{\varepsilon}}\right)^{i-1} \mathbf{B}_{\varepsilon}\right] \mathrm{d} A, \\
& \mathbf{K}_{\mu x 1}=l^{2} \int_{A}\left(\mathbf{B}_{x 1}\right)^{T}\left[(1-D)^{i-1} \mathbf{C}_{\sigma \varepsilon} \mathbf{B}_{\eta 1}-\mathbf{C}_{\sigma \varepsilon} \boldsymbol{\varepsilon}_{x 1}^{i-1}\left(\frac{\mathrm{d} D}{\mathrm{~d} \boldsymbol{\varepsilon}}\right)^{i-1} \mathbf{B}_{\varepsilon}\right] \mathrm{d} A, \\
& \mathbf{K}_{\mu x 2}=l^{2} \int_{A}\left(\mathbf{B}_{x 2}\right)^{T}\left[(1-D)^{i-1} \mathbf{C}_{\sigma \varepsilon} \mathbf{B}_{\eta 2}-\mathbf{C}_{\sigma \varepsilon} \boldsymbol{\varepsilon}_{x 2} i-1\left(\frac{\mathrm{d} D}{\mathrm{~d} \boldsymbol{\varepsilon}}\right)^{i-1} \mathbf{B}_{\varepsilon}\right] \mathrm{d} A .
\end{aligned}
$$

On the right-hand side of Eq. (16), the vectors $\mathbf{F}_{\mathrm{e}}$ and $\mathbf{F}_{\mathrm{i}}$ stand for the external and internal forces, respectively. The derived algorithm is embedded into the FE software ABAQUS [37] via user subroutines.

In the constitutive modelling of softening, the linear and exponential softening laws are usually used, as defined in [38]. The linear softening is expressed as

$$
D=\left\{\begin{array}{llc}
\frac{\kappa_{\mathrm{u}}\left(\kappa-\kappa_{0}\right)}{\kappa\left(\kappa_{\mathrm{u}}-\kappa_{0}\right)} & \text { if } & \kappa_{0} \leq \kappa \leq \kappa_{\mathrm{u}}, \\
1 & \text { if } & \kappa>\kappa_{\mathrm{u}},
\end{array}\right.
$$

while the exponential softening laws may be described in the following form

$$
D=1-\frac{\kappa_{0}}{\kappa}\left\{1-\alpha+\alpha \exp \left[\beta\left(\kappa_{0}-\kappa\right)\right]\right\} \text { if } \kappa>\kappa_{0} .
$$

Therein, scalar $\kappa$ is the history parameter, i.e., the highest value of the equivalent strain $\varepsilon_{\text {eq }}$ in the loading history. $\kappa_{\mathrm{u}}$ is the material parameter representing total stiffness loss, while $\kappa_{0}$ 
is the threshold value for initiation of the softening. The parameter $\alpha$ represents the stress decrease rate and $\beta$ is the damage rate.

For determination of the equivalent strain $\varepsilon_{\text {eq }}$, the Mazars' equivalent strain measure according to [39] written as

$$
\varepsilon_{\mathrm{eq}}=\sqrt{\sum_{i=1}^{3}\left\langle\varepsilon_{i}\right\rangle^{2}}
$$

is usually used. Here $\varepsilon_{i}(i=1,2,3)$ represents positive contribution of the principal strains. Besides, the following expression

$$
\varepsilon_{\text {eq }}=\frac{k-1}{2 k(1-2 v)} I_{1}+\frac{1}{2 k} \sqrt{\left(\frac{k-1}{1-2 v} I_{1}\right)^{2}+\frac{12 k}{(1+v)^{2}} J_{2}},
$$

based on the modified von Mises' definition may also be applied. Here, the parameter $k$ is the ratio of compressive and tensile strength, while $I_{1}$ and $J_{2}$ are the first strain tensor invariant and the second invariant of strain deviator, respectively [40].

\subsection{Nonlocal multiscale scheme for quasi-brittle damage analysis}

Here the $C^{1}-C^{1}$ multiscale scheme given in Fig. 2 is briefly described. The $C^{1}$ finite element presented in Section 2.1 is utilized for discretization at both scales. Starting from the global macrolevel BVP expressed by $\mathbf{K} \Delta \mathbf{V}=\mathbf{F}_{\mathrm{e}}-\mathbf{F}_{\mathrm{i}}$, the global DOF vector $\Delta \mathbf{V}$ is used for the determination of the local DOF vector $\Delta \mathbf{v}$ at single element level. Here the nonlocal finite element formulation employing only linear elastic material behaviour without damage derived in [7] is applied. By computing the macrostrain increment $\Delta \boldsymbol{\varepsilon}_{\mathrm{M}}$ and increment of the macrostrain gradient $\nabla \otimes \Delta \boldsymbol{\varepsilon}_{\mathrm{M}}$ in a macroscale material point, the increment of the MVE boundary displacements $\Delta \mathbf{u}_{\mathrm{b}}$ is formulated through boundary conditions. 


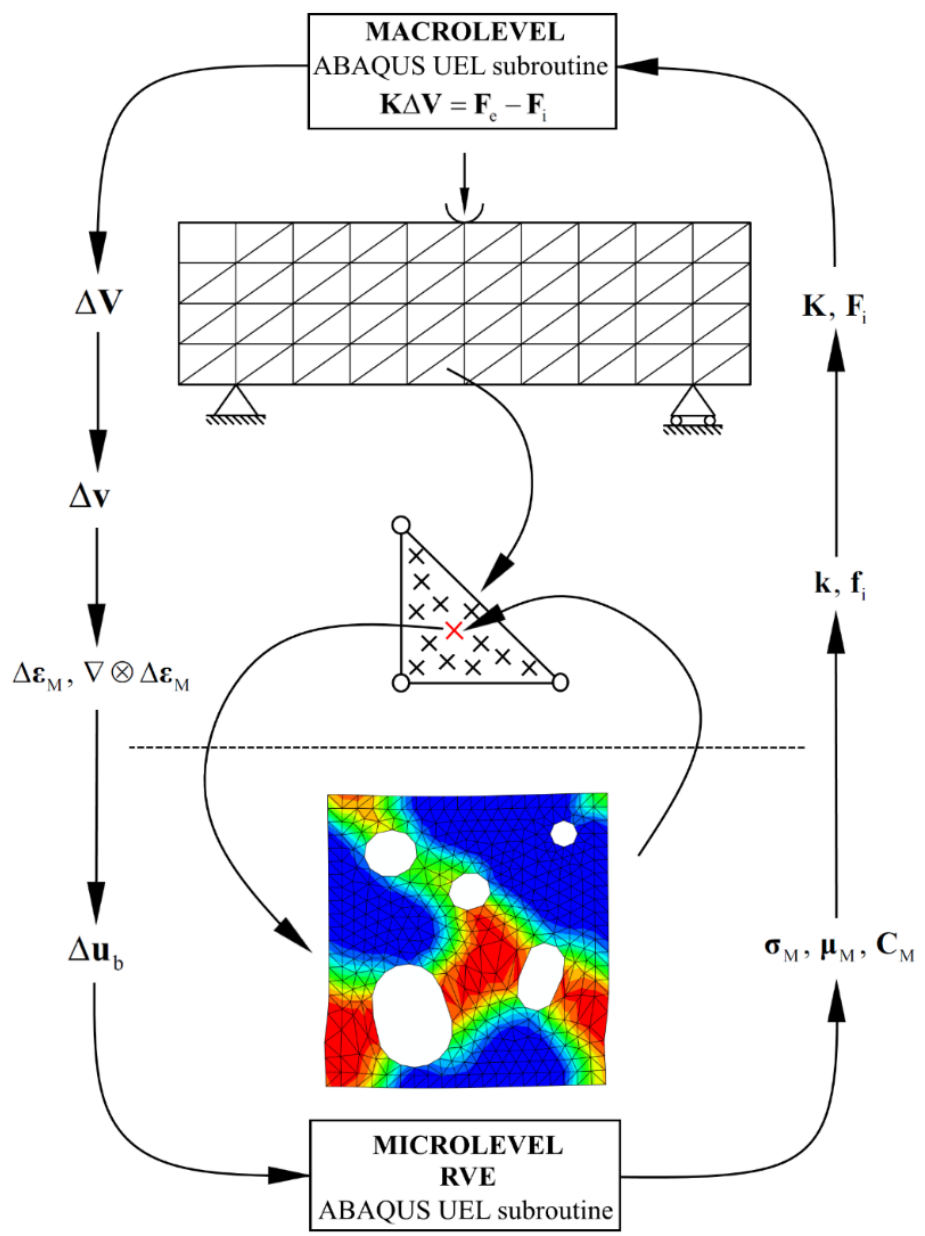

Fig. 2 Scheme of $C^{1}-C^{1}$ multiscale algorithm [36]

The microscale BVP is solved afterwards, where through the homogenization scheme the macrolevel constitutive response is obtained. For clarity reasons, the macroscopic quantities have the subscript "M", while the micro terms are labelled by the subscript "m". In the most general case, the Cauchy stress tensor $\boldsymbol{\sigma}_{M}$, the double stress tensor $\boldsymbol{\mu}_{M}$ and the constitutive tensor $\mathbf{C}_{\mathrm{M}}$ has to be homogenized. Once computed, the homogenized variables are upscaled, where they contribute to the formation of macrolevel stiffness and internal forces in a single material point. When the homogenized response is obtained throughout complete macroscale model, the global equation system is formed, and the updated macroscale BVP is defined. The procedure is repeated in the loop up to the convergence of the macrolevel BVP. Same routine used in this multiscale computational scheme has already been explained in more detail in [7], where the linear-elastic material behaviour without softening phenomena has been considered.

\subsection{Basic macro-micro scale transition relations}

The MVE boundary displacement field is expressed in terms of the macrolevel strain $\boldsymbol{\varepsilon}_{M}$ and the macrolevel strain gradient strain $\nabla \otimes \Delta \boldsymbol{\varepsilon}_{\mathrm{M}}$ by using the Taylor series expansion as

$$
\mathbf{u}_{\mathrm{b}}=\boldsymbol{\varepsilon}_{\mathrm{M}} \cdot \mathbf{x}+\frac{1}{2}\left[\mathbf{x} \cdot\left(\nabla \otimes \boldsymbol{\varepsilon}_{\mathrm{M}}\right) \cdot \mathbf{x}\right]+\mathbf{r}
$$


where $\mathbf{x}$ is the local microlevel coordinate, while $\mathbf{r}$ represents the microfluctuations [41]. The periodic boundary conditions are applied. More details about the micro-macro transition can be found in [7]. The scale transition scheme is based on the Hill-Mandel principle, i.e., work variation at the MVE corresponds to the work variation at the single macroscale point. Accordingly, the homogenized stresses are

$$
\begin{aligned}
& \boldsymbol{\sigma}_{\mathrm{M}}=\frac{1}{V} \int_{V} \boldsymbol{\sigma}_{\mathrm{m}} \mathrm{d} V, \\
& \boldsymbol{\mu}_{\mathrm{M}}=\frac{1}{V} \int_{V}\left(\boldsymbol{\mu}_{\mathrm{m}}+\boldsymbol{\sigma}_{\mathrm{m}} \otimes \mathbf{x}\right) \mathrm{d} V,
\end{aligned}
$$

where $V$ represents the MVE volume.

The following macroscopic constitutive relations are assumed

$$
\begin{aligned}
& \Delta \boldsymbol{\sigma}=\mathbf{C}_{\sigma \varepsilon}: \Delta \boldsymbol{\varepsilon}+\mathbf{C}_{\sigma \varepsilon x 1} \vdots \Delta \boldsymbol{\varepsilon}_{x 1}+\mathbf{C}_{\sigma \varepsilon x 2} \vdots \Delta \boldsymbol{\varepsilon}_{x 2}, \\
& \Delta \boldsymbol{\mu}_{x 1}=\mathbf{C}_{\mu x 1 \varepsilon}: \Delta \boldsymbol{\varepsilon}+\mathbf{C}_{\mu x 1 \varepsilon x 1} \vdots \Delta \boldsymbol{\varepsilon}_{x 1}+\mathbf{C}_{\mu x 1 \varepsilon x 2} \vdots \Delta \boldsymbol{\varepsilon}_{x 2}, \\
& \Delta \boldsymbol{\mu}_{x 2}=\mathbf{C}_{\mu x 2 \varepsilon}: \Delta \boldsymbol{\varepsilon}+\mathbf{C}_{\mu x 2 \varepsilon x 1} \vdots \Delta \boldsymbol{\varepsilon}_{x 1}+\mathbf{C}_{\mu x 2 \varepsilon x 2} \vdots \Delta \boldsymbol{\varepsilon}_{x 2} .
\end{aligned}
$$

These relations contain nine constitutive matrices, giving possibility to capture the contributions of heterogeneities and microstructural interactions,

$$
\begin{aligned}
& \mathbf{C}_{\sigma \varepsilon}=\frac{1}{V} \mathbf{D} \tilde{\mathbf{K}}_{\mathrm{bb}} \mathbf{D}^{T}, \quad \mathbf{C}_{\sigma \varepsilon x 1}=\frac{1}{V} \mathbf{D} \tilde{\mathbf{K}}_{\mathrm{bb}} \mathbf{H}_{1}^{T}, \quad \mathbf{C}_{\sigma \varepsilon x 2}=\frac{1}{V} \mathbf{D} \tilde{\mathbf{K}}_{\mathrm{bb}} \mathbf{H}_{2}^{T}, \\
& \mathbf{C}_{\mu x 1 \varepsilon}=\frac{1}{V} \mathbf{H}_{1} \tilde{\mathbf{K}}_{\mathrm{bb}} \mathbf{D}^{T}, \quad \mathbf{C}_{\mu x 1 \varepsilon x 1}=\frac{1}{V} \mathbf{H}_{1} \tilde{\mathbf{K}}_{\mathrm{bb}} \mathbf{H}_{1}^{T}, \quad \mathbf{C}_{\mu x 1 \varepsilon x 2}=\frac{1}{V} \mathbf{H}_{1} \tilde{\mathbf{K}}_{\mathrm{bb}} \mathbf{H}_{2}^{T}, \\
& \mathbf{C}_{\mu x 2 \varepsilon}=\frac{1}{V} \mathbf{H}_{2} \tilde{\mathbf{K}}_{\mathrm{bb}} \mathbf{D}^{T}, \quad \mathbf{C}_{\mu x 2 \varepsilon x 1}=\frac{1}{V} \mathbf{H}_{2} \tilde{\mathbf{K}}_{\mathrm{bb}} \mathbf{H}_{1}^{T}, \quad \mathbf{C}_{\mu x 2 \varepsilon x 2}=\frac{1}{V} \mathbf{H}_{2} \tilde{\mathbf{K}}_{\mathrm{bb}} \mathbf{H}_{2}^{T} .
\end{aligned}
$$

Here $\mathbf{D}, \mathbf{H}_{1}$ and $\mathbf{H}_{2}$ represent the coordinate matrices, and their detailed derivation can be found in [42]. $\tilde{\mathbf{K}}_{\mathrm{bb}}$ is the condensed MVE stiffness matrix, as shown in [41], and it is obtained here by Eqs. (17) - (19). Since the element stiffness is related to the damage variable $D$, the damage is implicitly included into the condensed MVE stiffness matrix, i.e.

$$
\tilde{\mathbf{K}}_{\mathrm{bb}}=\tilde{\mathbf{K}}_{\mathrm{bb}}(D) \text {. }
$$

Generally, in the homogenization, the macrolevel constitutive operators are derived from the condensed microlevel stiffness,

$$
\mathbf{C}_{\mathrm{M}}=\mathbf{C}_{\mathrm{M}}\left(\tilde{\mathbf{K}}_{\mathrm{bb}}\right) .
$$

From (31) and (32) it is clear that the homogenized constitutive matrix is dependent on the microstructural damage. Accordingly, with the localization in the MVE the associated macrolevel material point is losing load-carrying capability. The crack forms at this material point and the stiffness values are kept close to zero in the numerical model. More on the MVE failure condition is discussed in [36]. 


\subsection{Numerical example. Plate subjected to tensile load}

As an example, the plate with an imperfection is loaded along the right edge. The computational model is shown in Fig. 3, discretized by 48 triangular finite elements, as depicted in Fig. 4. This relatively coarse mesh is used after convergence and mesh sensitivity analysis performed in [36].

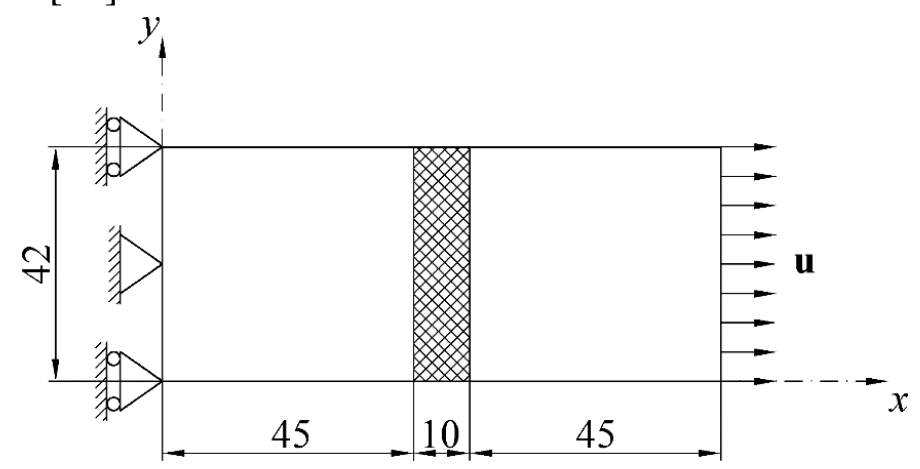

Fig. 3 Geometry and boundary conditions

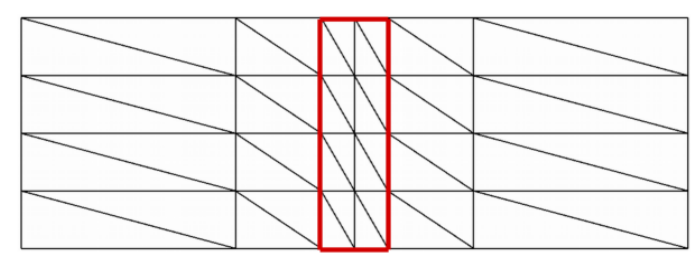

Fig. 4 Macrolevel discretization consisting of $48 C^{1}$ continuity triangular finite elements

Mazars' equivalent strain defined in (22) is used with the linear softening law given in (20). The heterogeneous microstructure described by an academic MVE presented in Fig. 5 has the following material properties: $E=20000 \mathrm{~N} / \mathrm{mm}^{2}, \quad v=0.25, \quad \kappa_{0}=0.0001$, $\kappa_{\mathrm{u}}=0.0125$. At the right edge, displacement $u=0.0325 \mathrm{~mm}$ is imposed (Fig. 3). To initiate softening, the Young's modulus is $10 \%$ reduced in the middle hatched area. At the left and the right edge, the second derivatives $u_{1,11}, u_{1,22}, u_{1,12}, u_{2,12}$ and the first derivatives $u_{1,2}$ and $u_{2,1}$ are suppressed, yielding straight edges.

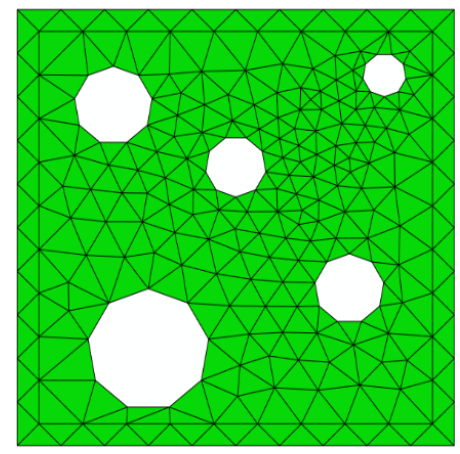

Fig. 5 MVE discretization by $C^{1}$ continuity triangular finite elements

The length scale parameter is $l=0.025 \mathrm{~mm}$, and the MVE side length is $L=2.6 \mathrm{~mm}$. Porosity ratio is $e=0.13$ with average hole radius $r_{\text {ave }}=0.559 \mathrm{~mm}$.

The distribution of the longitudinal strain at the stage of softening initiation is shown in Fig. 6. Due to relatively coarse mesh discretization, smooth contour visualization is hard to 
get. But, even with a sparse mesh it is visible that the localization band is forming in a physically acceptable way.

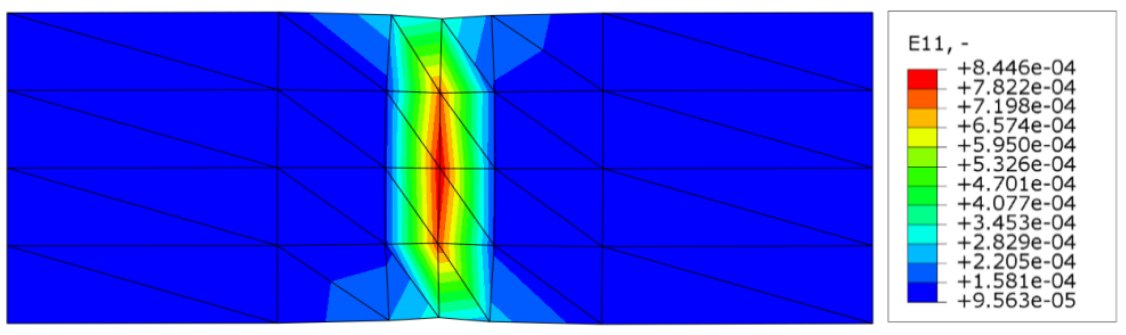

Fig. 6 Distribution of strain component $\varepsilon_{11}$ at the moment of macromodel softening initiation

The contour plot of the damage zones at the MVEs appointed to the characteristic macrostructural points within and near the localization band is shown in Fig. 7. As can be seen, the most intense microstructural softening is found in the material points in the middle of the plate. Therefore, it can be concluded that the numerical results can be taken as realistic at both scales.

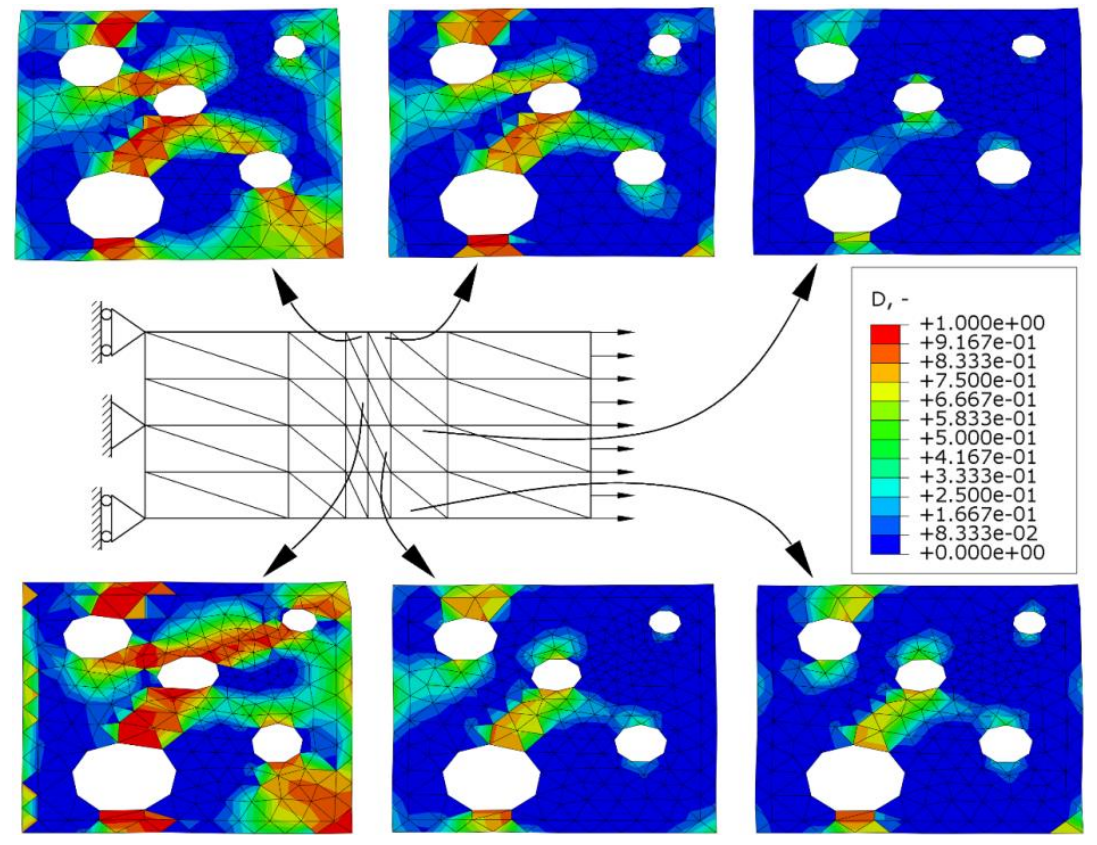

Fig. 7 Distribution of microscale damage at the moment of softening initiation in macromodel [36]

\section{Ductile damage modelling of heterogeneous material}

\subsection{Macrolevel finite element formulation}

As in the formulation presented above, the second-order homogenization scheme relies on gradient theory application at the macroscale. Hence, the macroscale is discretized by the plane strain $C^{1}$ finite element already presented in Fig. 1.

The relevant element matrices are derived from the virtual work principle

$$
\int_{A} \delta \boldsymbol{\varepsilon}^{T} \boldsymbol{\sigma} \mathrm{d} A+\int_{A} \delta \boldsymbol{\eta}^{T} \boldsymbol{\mu} \mathrm{d} A=\int_{S} \delta \mathbf{u}^{T} \mathbf{t} \mathrm{d} s+\int_{S} \delta\left(\operatorname{grad} \mathbf{u}^{T}\right) \mathbf{T} \mathrm{d} s .
$$

In (33), $\boldsymbol{\sigma}$ and $\boldsymbol{\varepsilon}$ represent the stress and strain tensors, while $\boldsymbol{\mu}$ and $\boldsymbol{\eta}$ represent the double stress and strain tensors. The displacement vector is denoted by $\mathbf{u}, \mathbf{t}$ is the surface traction, while $\mathbf{T}$ is the double surface traction, respectively. The integration of the variables is 
performed over the element surface $A$ and outside boundary $s$. Through the discretization procedure the strains are expressed by DOF as

$$
\boldsymbol{\varepsilon}=\left[\begin{array}{c}
\varepsilon_{11} \\
\varepsilon_{22} \\
2 \varepsilon_{12}
\end{array}\right]=\mathbf{B}_{\varepsilon} \mathbf{v}, \boldsymbol{\eta}=\left[\begin{array}{c}
\eta_{111} \\
\eta_{222} \\
\eta_{221} \\
\eta_{112} \\
2 \eta_{121} \\
2 \eta_{212}
\end{array}\right]=\left[\begin{array}{c}
u_{1,11} \\
u_{2,22} \\
u_{1,22} \\
u_{2,11} \\
2 u_{1,21} \\
2 u_{2,12}
\end{array}\right]=\mathbf{B}_{\eta} \mathbf{v} .
$$

$\mathbf{B}_{\varepsilon}$ and $\mathbf{B}_{\eta}$ matrices consist of first- and second shape function derivatives. Since the homogenization concept relies on no a priori assumptions on the constitutive behaviour, the updates of the stresses are dependent on both the strain and the double strain

$$
\begin{aligned}
\Delta \boldsymbol{\sigma} & =\mathbf{C}_{\sigma \varepsilon} \Delta \boldsymbol{\varepsilon}+\mathbf{C}_{\sigma \eta} \Delta \boldsymbol{\eta}, \\
\Delta \boldsymbol{\mu} & =\mathbf{C}_{\mu \varepsilon} \Delta \boldsymbol{\varepsilon}+\mathbf{C}_{\mu \eta} \Delta \boldsymbol{\eta} .
\end{aligned}
$$

The constitutive operators $\mathbf{C}_{\sigma \varepsilon}, \mathbf{C}_{\sigma \eta}, \mathbf{C}_{\mu \varepsilon}$ and $\mathbf{C}_{\mu \eta}$ are obtained by the homogenization at the MVE.

By means of (33) - (35), the usual nonlinear FE equation is derived $\left(\mathbf{K} \Delta \mathbf{v}=\mathbf{F}_{\mathrm{e}}-\mathbf{F}_{\mathrm{i}}\right)$. The stiffness matrix $\mathbf{K}$ consists of

$$
\mathbf{K}=\mathbf{K}_{\sigma \varepsilon}+\mathbf{K}_{\sigma \eta}+\mathbf{K}_{\mu \varepsilon}+\mathbf{K}_{\mu \eta}
$$

where the stiffness submatrices are

$$
\begin{aligned}
& \mathbf{K}_{\sigma \varepsilon}=\int_{A}\left(\mathbf{B}_{\varepsilon}^{T} \mathbf{C}_{\sigma \varepsilon} \mathbf{B}_{\varepsilon}\right) \mathrm{d} A, \\
& \mathbf{K}_{\sigma \eta}=\int_{A}\left(\mathbf{B}_{\varepsilon}^{T} \mathbf{C}_{\sigma \eta} \mathbf{B}_{\eta}\right) \mathrm{d} A, \\
& \mathbf{K}_{\mu \varepsilon}=\int_{A}\left(\mathbf{B}_{\eta}^{T} \mathbf{C}_{\mu \varepsilon} \mathbf{B}_{\varepsilon}\right) \mathrm{d} A, \\
& \mathbf{K}_{\mu \eta}=\int_{A}\left(\mathbf{B}_{\eta}^{T} \mathbf{C}_{\mu \eta} \mathbf{B}_{\eta}\right) \mathrm{d} A .
\end{aligned}
$$

The vectors $\mathbf{F}_{\mathrm{e}}$ and $\mathbf{F}_{\mathrm{i}}$ represent the nodal forces (external and internal)

$$
\begin{aligned}
& \mathbf{F}_{\mathrm{e}}=\int_{s}\left(\mathbf{N}^{T} \mathbf{t}+\operatorname{grad} \mathbf{N}^{T} \mathbf{T}\right) \mathrm{d} s, \\
& \mathbf{F}_{\mathrm{i}}=\int_{A}\left(\mathbf{B}_{\varepsilon}^{T} \boldsymbol{\sigma}^{i-1}+\mathbf{B}_{\eta}^{T} \boldsymbol{\mu}^{i-1}\right) \mathrm{d} A .
\end{aligned}
$$

The derivation of all the relations presented above are in more detail described in [41]. 


\subsection{Nonlocal ductile damage model and computational homogenization}

The ductile softening model in combination with the computational homogenization is employed at the MVE. In contrast to the quasi-brittle damage modelling, here the MVE is discretized by mixed quadrilateral finite elements, and the $C^{1}-C^{0}$ transition procedure is applied.

To capture ductile damage, the gradient-enhanced elastoplasticity employing damage evolution law is proposed [43]. The yield function is

$$
F\left(\boldsymbol{\sigma}, \varepsilon_{\mathrm{p}}, D\right)=\sigma_{\mathrm{e}}(\boldsymbol{\sigma})-(1-D) \sigma_{y}\left(\varepsilon_{\mathrm{p}}\right)
$$

where $\sigma_{\mathrm{e}}$ is the von Mises stress, while $\varepsilon_{\mathrm{p}}$ is the standard equivalent plastic strain. The damage evolution is described by

$$
D\left(\bar{\varepsilon}_{\mathrm{p}}\right)=1-e^{-\beta \bar{\varepsilon}_{\mathrm{p}}} \text {. }
$$

Herein $\beta$ is the material parameter, and $\bar{\varepsilon}_{\mathrm{p}}$ represents the nonlocal equivalent plastic strain.

Two differential equations are simultaneously solved, the standard equilibrium equation, and the Helmholtz type equation with the microstructural parameter $l^{2}$ written as

$$
\bar{\varepsilon}_{\mathrm{p}}-l^{2} \nabla^{2} \bar{\varepsilon}_{\mathrm{p}}=\varepsilon_{\mathrm{p}} .
$$

The aforementioned equations are embedded into the $C^{0}$ linear quadrilateral finite element for plane strain problems, with $\bar{\varepsilon}_{\mathrm{p}}$ as an additional nodal variable. As explained in more detail in [35], the following FE equation is obtained

$$
\left[\begin{array}{cc}
\mathbf{K}_{u u} & \mathbf{K}_{u \bar{\varepsilon}_{\mathrm{p}}} \\
\mathbf{K}_{\bar{\varepsilon}_{\mathrm{p}} u} & \mathbf{K}_{\bar{\varepsilon}_{\mathrm{p}} \bar{\varepsilon}_{\mathrm{p}}}
\end{array}\right]\left[\begin{array}{c}
\Delta \mathbf{u} \\
\Delta \bar{\varepsilon}_{\mathrm{p}}
\end{array}\right]=\left[\begin{array}{c}
\mathbf{F}_{\mathrm{e}}^{u}-\mathbf{F}_{\mathrm{i}}^{u} \\
\mathbf{F}_{\mathrm{i}}^{\bar{\varepsilon}_{\mathrm{p}}}
\end{array}\right],
$$

where $\mathbf{K}_{u u}, \mathbf{K}_{u \bar{\varepsilon}_{\mathrm{p}}}, \mathbf{K}_{\bar{\varepsilon}_{\mathrm{p}} u}, \mathbf{K}_{\bar{\varepsilon}_{\mathrm{p}} \bar{\varepsilon}_{\mathrm{p}}}$ are the stiffness submatrices. $\mathbf{F}_{\mathrm{e}}^{u}$ is the displacement-based external loading vector, while $\mathbf{F}_{\mathrm{i}}^{u}$ and $\mathbf{F}_{\mathrm{i}}{ }^{\bar{\varepsilon}_{\mathrm{p}}}$ are the internal forces due to the displacements and nonlocal plastic strains.

For the homogenization purposes, from the equation system (42), the following relation is extracted [7, 44]

$$
\mathbf{K}_{u u} \Delta \mathbf{u}=\Delta \mathbf{f}^{u},
$$

which is then partitioned into

$$
\left[\begin{array}{ll}
\mathbf{K}_{\mathrm{aa}} & \mathbf{K}_{\mathrm{ab}} \\
\mathbf{K}_{\mathrm{ba}} & \mathbf{K}_{\mathrm{bb}}
\end{array}\right]\left[\begin{array}{c}
\Delta \mathbf{u}_{\mathrm{a}} \\
\Delta \mathbf{u}_{\mathrm{b}}
\end{array}\right]=\left[\begin{array}{c}
\Delta \mathbf{f}_{\mathrm{a}} \\
\Delta \mathbf{f}_{\mathrm{b}}
\end{array}\right] .
$$

Here $\Delta \mathbf{f}_{\mathrm{b}}$ contains the nodal forces at the outside boundary of the MVE, while $\Delta \mathbf{f}_{\mathrm{a}}$ are the nodal forces at the interior of the MVE vanishing at convergence.

From (44), the condensed MVE stiffness matrix can be found

$$
\tilde{\mathbf{K}}_{\mathrm{bb}}=\mathbf{K}_{\mathrm{bb}}-\mathbf{K}_{\mathrm{ba}} \mathbf{K}_{\mathrm{aa}}^{-1} \mathbf{K}_{\mathrm{ab}},
$$


which is necessary for the macrolevel constitutive relations. The updates of the stress tensors can be written as

$$
\begin{aligned}
& \Delta \boldsymbol{\sigma}_{\mathrm{M}}=\frac{1}{V}\left(\mathbf{D} \tilde{\mathbf{K}}_{\mathrm{bb}} \mathbf{D}^{T} \Delta \boldsymbol{\varepsilon}_{\mathrm{M}}+\mathbf{D} \tilde{\mathbf{K}}_{\mathrm{bb}} \mathbf{H}^{T} \Delta \boldsymbol{\eta}_{\mathrm{M}}\right), \\
& \Delta \boldsymbol{\mu}_{\mathrm{M}}=\frac{1}{V}\left(\mathbf{H} \tilde{\mathbf{K}}_{\mathrm{bb}} \mathbf{D}^{T} \Delta \boldsymbol{\varepsilon}_{\mathrm{M}}+\mathbf{H} \tilde{\mathbf{K}}_{\mathrm{bb}} \mathbf{H}^{T} \Delta \boldsymbol{\eta}_{\mathrm{M}}\right),
\end{aligned}
$$

that leads to the macroscale constitutive matrices

$$
\begin{aligned}
& \mathbf{C}_{\sigma \varepsilon}=\frac{1}{V} \mathbf{D} \tilde{\mathbf{K}}_{\mathrm{bb}} \mathbf{D}^{T}, \\
& \mathbf{C}_{\sigma \eta}=\frac{1}{V} \mathbf{D} \tilde{\mathbf{K}}_{\mathrm{bb}} \mathbf{H}^{T}, \\
& \mathbf{C}_{\mu \varepsilon}=\frac{1}{V} \mathbf{H} \tilde{\mathbf{K}}_{\mathrm{bb}} \mathbf{D}^{T}, \\
& \mathbf{C}_{\mu \eta}=\frac{1}{V} \mathbf{H} \tilde{\mathbf{K}}_{\mathrm{bb}} \mathbf{H}^{T} .
\end{aligned}
$$

Herein, $\mathbf{D}$ and $\mathbf{H}$ are the MVE coordinate matrices, as described in [42]. Since the condensed stiffness $\tilde{\mathbf{K}}_{\mathrm{bb}}$ depends on the damage and microstructural scale parameter, $\tilde{\mathbf{K}}_{\mathrm{bb}}=\tilde{\mathbf{K}}_{\mathrm{bb}}(D, l)$, the damage is upscaled through the homogenized constitutive operators (47).

The presented formulations and procedures have been embedded into the FE software ABAQUS.

\subsection{Scale transition approach}

Analogous to the two-scale computational procedure discussed in the previous sections where the quasi-brittle damage is considered, every macrolevel material point has the MVE appointed in which the solution of BVP governs the macroscale material response. Analogous to the computational scheme shown in Fig. 2, from the displacement increment $\Delta \mathbf{v}$, displacement gradients $\Delta \boldsymbol{\varepsilon}_{\mathrm{M}}$ and $\Delta \boldsymbol{\eta}_{\mathrm{M}}$ are computed and used for the computation of the MVE boundary displacement increment $\Delta \mathbf{u}_{\mathrm{b}}$

$$
\Delta \mathbf{u}_{\mathrm{b}}=\mathbf{D}^{T} \boldsymbol{\varepsilon}_{\mathrm{M}}+\mathbf{H}^{T} \boldsymbol{\eta}_{\mathrm{M}},
$$

assuming periodic boundary conditions. As mentioned already, in the conventional homogenization the averaging of stresses is performed through entire MVE volume. Accordingly, the macrolevel stress tensors are expressed as

$$
\begin{aligned}
& \boldsymbol{\sigma}_{\mathrm{M}}=\frac{1}{V} \int_{V} \boldsymbol{\sigma}_{\mathrm{m}} \mathrm{d} V, \\
& \boldsymbol{\mu}_{\mathrm{M}}=\frac{1}{2 V} \int_{V}\left(\boldsymbol{\sigma}_{\mathrm{m}}^{T} \mathbf{x}+\mathbf{x}^{T} \boldsymbol{\sigma}_{\mathrm{m}}\right) \mathrm{d} V .
\end{aligned}
$$

For determination of the homogenized constitutive response, the static condensation procedure is conducted, as explained in the previous section. The simple transition scheme is graphically depicted in Fig. 8. 


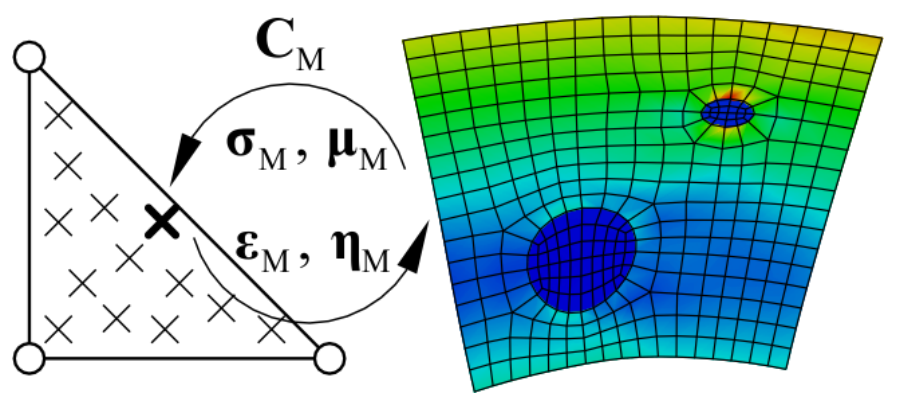

Fig. 8 Scheme of the scale transition

As already explained, the averaged microstructural quantities are expressed in dependency to damage, providing softening at the macrolevel. In the case of severe softening at corresponding macroscale material point, its stiffness is set to small magnitude, preserving numerical stability of the macromodel. The softening criterion and determination of the microscale localization zone is more discussed in $[35,45]$.

\subsection{Numerical examples}

\subsubsection{Strip subjected to tensile loading}

As in the previous example, a tensile loaded heterogeneous strip is considered. The geometry of the macromodel with boundary conditions remains unchanged as presented in Fig 3. The strip is discretized by 42 finite elements, as shown in Fig. 9.

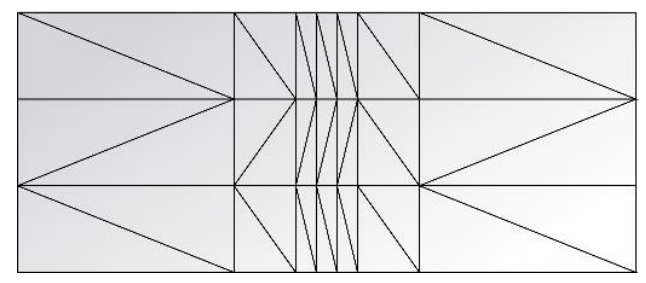

Fig. 9 Strip discretization

The weakened zone is again placed in the middle area, where the yield stress is kept lower to initiate softening. The MVE size is $L=0.2 \mathrm{~mm}$, with $13 \%$ of porosity ratio and average void radius $0.043 \mathrm{~mm}$. Young's modulus of the matrix is $210 \mathrm{GPa}$ with Poisson's ratio 0.3. Linear isotropic strain hardening with the modulus $h=20000 \mathrm{MPa}$ is exhibited at the yield stress of $250 \mathrm{MPa}$. The softening law is expressed in (40), with the microstructural parameter $l=0.1 \mathrm{~mm}$ and the softening exponent $\beta=200$. The yield stress in the weakened zone is set to $240 \mathrm{MPa}$. The MVE is discretized by 508 finite elements, as shown in Fig. 10.

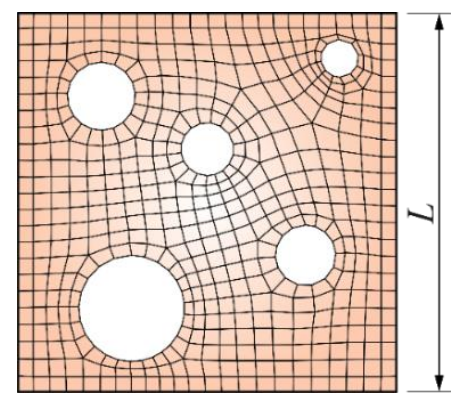

Fig. 10 RVE quadrilateral finite element discretization 
The distribution of damage contours at both scales at the complete collapse is presented in Fig. 11. The collapse has occurred in logical sense within the weakened zone at the macroscale, which is also observable through the damage distribution on the MVEs.
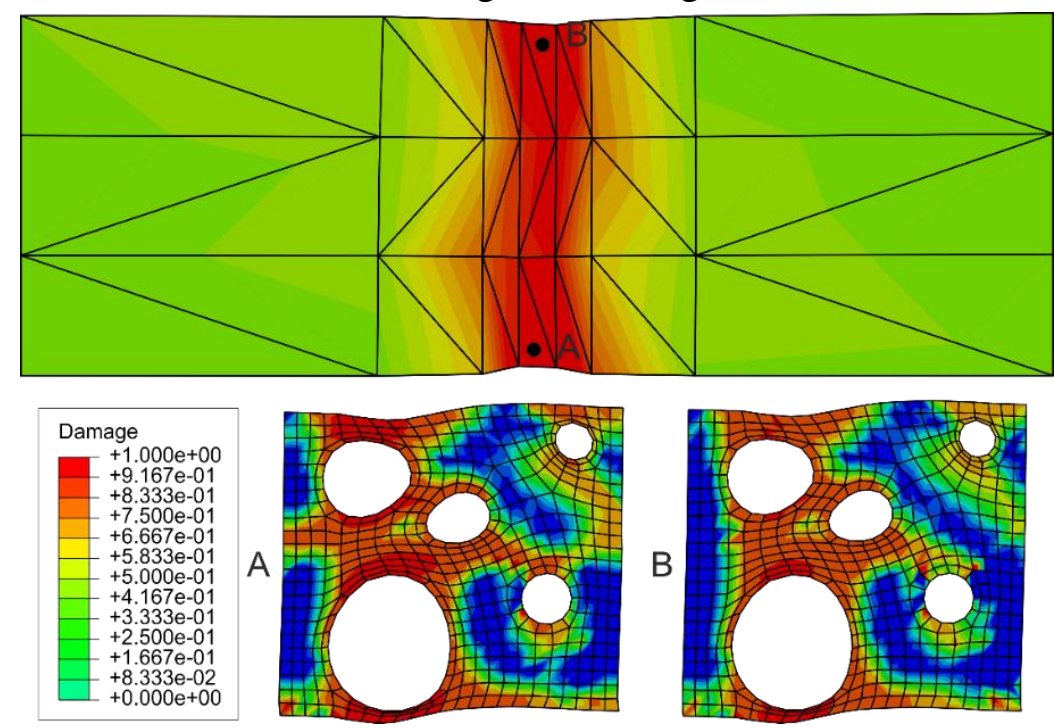

Fig. 11 Micro- and macrolevel damage distribution at the structural collapse

\subsubsection{Plate subjected to compressive loading}

The second example dealing with ductile damage is a plate with compressive loading. The macrolevel model discretization is presented in Fig. 12. The horizontal edges of the plate are modelled as straight lines. The geometry parameter $H$ is $50 \mathrm{~mm}$. The softening is initiated in the middle zone of the size $0.1 H \times 0.1 H$ by setting yield stress to a lower value. The MVE and material used are equal as in the previous example.

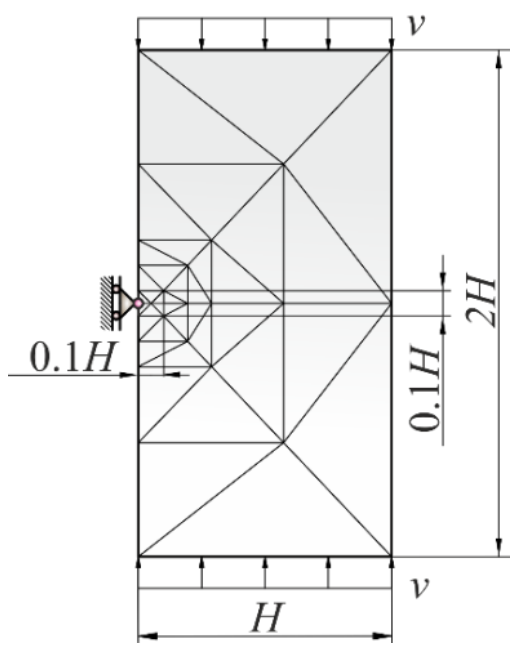

Fig. 12 Plate subjected to compression

The damage distribution on both scales at the total collapse is displayed in Fig. 13. 
Jurica Sorić, Tomislav Lesičar Filip Putar, Zdenko Tonković
On Multiscale Damage Modeling of Heterogeneous Materials Using Nonlocal Continuum Theory

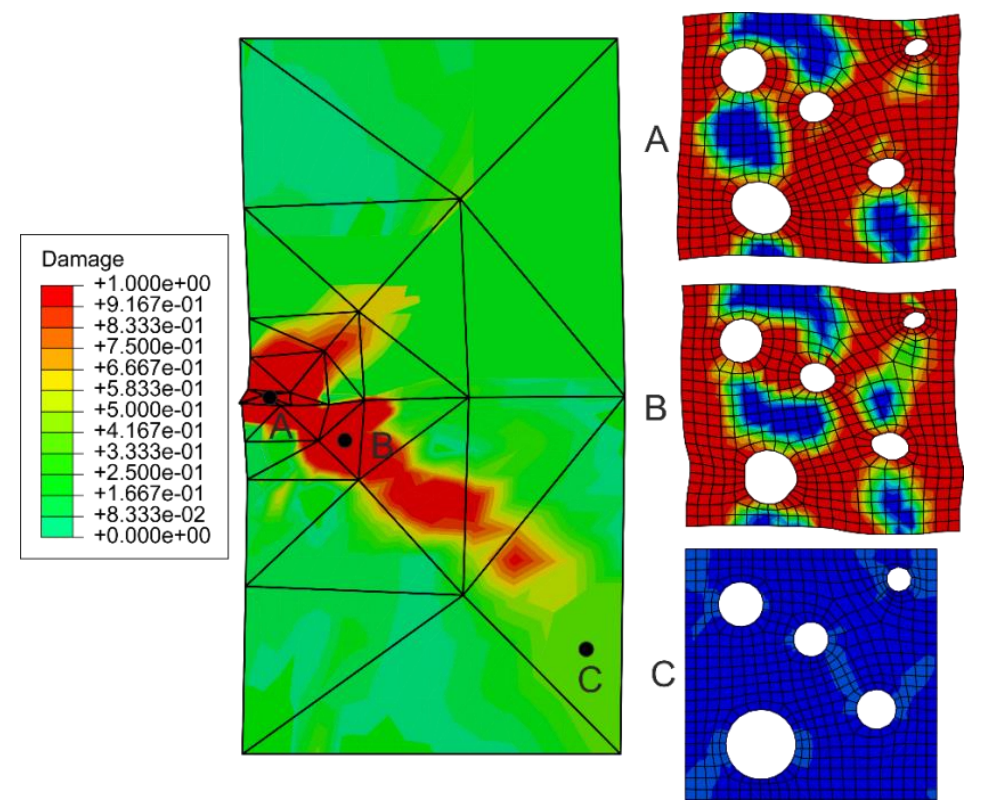

Fig. 13 Damage distribution at the structural collapse

After the weakness initiation, the localization band propagates towards the opposite outside edge, which confirms the expected realistic behaviour. The macrolevel response is confirmed by the damage contour distribution on the MVEs at points $\mathrm{A}, \mathrm{B}$ and $\mathrm{C}$. Both examples are also considered and discussed in [45].

Finally, it is useful to comment numerical effort and computational time of the multiscale schemes presented within this paper. The element formulation at the macroscale requires 13 Gauss points, where every material point has a MVE appointed. The homogenization procedure of a single MVE takes several seconds, depending on the MVE mesh discretization, where the most of computational time is consumed for condensation of the MVE stiffness. Since this procedure is repeated at every macrolevel material point, the computational time of any problem solved by utilization of multiscale scheme takes several hours, even days. This was the case also with the example problems solved in this paper. The computational efficiency can be significantly improved by parallelization, where multiple MVEs are homogenized at once. However, this is out of scope of this paper.

\section{Conclusion}

In the present paper an overview of the multiscale modelling of softening material behaviour including quasi-brittle and ductile damage is given. The formulation of the quasibrittle softening, employing the $C^{1}$ triangular finite elements incorporating the nonlocal continuum theory at macro and microlevel, is presented. The damage law is embedded into the constitutive response over the MVE. According to the multiscale strategy, the macrolevel localization is driven by the homogenized microlevel response.

The two-scale modelling of ductile softening by means of the second-order homogenization is also demonstrated. Therein, the macrolevel is discretized by the $C^{1}$ triangular finite element, while the microstructural softening is expressed through the implicit gradient-enhanced elastoplasticity embedded into the mixed quadrilateral finite element.

The numerical examples demonstrate the physical consistency of the softening at both scales. Despite the fact that the presented formulations deliver the results which are physically correct and realistic, the computations are complex and time demanding. Therefore, the computationally more efficient formulations are desirable. Further research is directed to the 
improving of computational efficiency and stability of computational homogenization in the frame of multiscale strategies, where advanced machine learning techniques [46] will be developed.

\section{REFERENCES}

[1] S. W. Kim, S. J. Lee, Development of a New Fatigue Damage Model For Quarter-Modal Spectra in Frequency Domain, Brodogradnja 71(1) (2020) 39-57. https://doi.org/10.21278/brod71103

[2] Q. Zhu, P. Zhang, X. Peng, L. Yan, G. Li, Fatigue Crack Growth Behavior and Fracture Toughness of EH36 TMCP Steel, Materials 14 (2021), 6621. https://doi.org/10.3390/ma14216621

[3] D. Perić, E.A. de Souza Neto, R.A. Feijóo, M. Partovi, A.J.C. Molina, On micro-to-macro transitions for multi-scale analysis of non-linear heterogeneous materials: unified variational basis and finite element implementation, Int. J. Numer. Methods Eng. 87 (2011) 149-170. https://doi.org/10.1002/nme.3014

[4] W.A.M. Brekelmans, M.G.D. Geers, V.G. Kouznetsova, Computational Homogenisation for Non-Linear Heterogeneous Solids, Multiscale Model. Solid Mech. (2009) 1-42. https://doi.org/10.1142/97818481630890001

[5] V.G. Kouznetsova, M.G.D. Geers, W.A.M. Brekelmans, Multi-scale second-order computational homogenization of multi-phase materials: a nested finite element solution strategy, Comput. Methods Appl. Mech. Eng. 193 (2004) 5525-5550. https://doi.org/10.1016/j.cma.2003.12.073

[6] V.D. Nguyen, G. Becker, L. Noels, Multiscale computational homogenization methods with a gradient enhanced scheme based on the discontinuous Galerkin formulation, Comput. Methods Appl. Mech. Eng. 260 (2013) 63-77. https://doi.org/10.1016/j.cma.2013.03.024

[7] T. Lesičar, Z. Tonković, J. Sorić, Two-scale computational approach using strain gradient theory at microlevel, Int. J. Mech. Sci. 126 (2017) 37-78. https://doi.org/10.1016/j.ijmecsci.2017.02.017

[8] T. Wu, İ. Temizer, P. Wriggers, Computational thermal homogenization of concrete, Cem. Concr. Compos. 35 (2013) 59-70. https://doi.org/10.1016/j.cemconcomp.2012.08.026

[9] C. Miehe, Computational micro-to-macro transitions for discretized micro-structures of heterogeneous materials at finite strains based on the minimization of averaged incremental energy, Comput. Methods Appl. Mech. Eng. 192 (2003) 559-591. https://doi.org/10.1016/S0045-7825(02)00564-9

[10] I. Gitman, H. Askes, E. Aifantis, The Representative Volume Size in Static and Dynamic Micro-Macro Transitions, Int. J. Fract. 135 (2005) L3-L9. https://doi.org/10.1007/s10704-005-4389-6

[11] I.M. Gitman, M.B. Gitman, H. Askes, Quantification of stochastically stable representative volumes for random heterogeneous materials, Arch. Appl. Mech. 75 (2006) 79-92. https://doi.org/10.1007/s00419005-0411-8

[12] S. Graham, N. Yang, Representative volumes of materials based on microstructural statistics, Scr. Mater. 48 (2003) 269-274. https://doi.org/10.1016/S1359-6462(02)00362-7

[13] I.M. Gitman, H. Askes, L.J. Sluys, Representative volume: Existence and size determination, Eng. Fract. Mech. 74 (2007) 2518-2534. https://doi.org/10.1016/j.engfracmech.2006.12.021

[14] I.M. Gitman, H. Askes, L.J. Sluys, Coupled-volume multi-scale modelling of quasi-brittle material, Eur. J. Mech. - A/Solids. 27 (2008) 302-327. https://doi.org/10.1016/j.euromechsol.2007.10.004

[15] T. Belytschko, S. Loehnert, J.-H. Song, Multiscale aggregating discontinuities: A method for circumventing loss of material stability, Int. J. Numer. Methods Eng. 73 (2008) 869-894. https://doi.org/10.1002/nme.2156

[16] T. Hettich, A. Hund, E. Ramm, Modeling of failure in composites by X-FEM and level sets within a multiscale framework, Comput. Methods Appl. Mech. Eng. 197 (2008) 414-424. https://doi.org/10.1016/j.cma.2007.07.017

[17] K. Matouš, M.G. Kulkarni, P.H. Geubelle, Multiscale cohesive failure modeling of heterogeneous adhesives, J. Mech. Phys. Solids. 56 (2008) 1511-1533. https://doi.org/10.1016/j.jmps.2007.08.005

[18] T.J. Massart, R.H.J. Peerlings, M.G.D. Geers, An enhanced multi-scale approach for masonry wall computations with localization of damage, Int. J. Numer. Methods Eng. 69 (2007) 1022-1059. https://doi.org/10.1002/nme.1799

[19] E. Bosco, V.G. Kouznetsova, E.W.C. Coenen, M.G.D. Geers, A. Salvadori, A multiscale framework for localizing microstructures towards the onset of macroscopic discontinuity, Comput. Mech. 54 (2014) 299-319. https://doi.org/10.1007/s00466-014-0986-4 
Jurica Sorić, Tomislav Lesičar

Filip Putar, Zdenko Tonković
On Multiscale Damage Modeling of Heterogeneous Materials Using Nonlocal Continuum Theory

[20] C. V Verhoosel, J.J.C. Remmers, M.A. Gutiérrez, R. de Borst, Computational homogenization for adhesive and cohesive failure in quasi-brittle solids, Int. J. Numer. Methods Eng. 83 (2010) 1155-1179. https://doi.org/10.1002/nme.2854

[21] L.J. Sluys, V.P. Nguyen, M. Stroeven, Multiscale Continuos and Discountinuous Modeling of Heterogeneous Materials: A Review on Recent Developments, J. Multiscale Model. 03-04 (2011) 229270. https://doi.org/10.1142/S1756973711000509

[22] V. Phu Nguyen, O. Lloberas-Valls, M. Stroeven, L. Johannes Sluys, On the existence of representative volumes for softening quasi-brittle materials - A failure zone averaging scheme, Comput. Methods Appl. Mech. Eng. 199 (2010) 3028-3038. https://doi.org/10.1016/j.cma.2010.06.018

[23] P.J. Sánchez, P.J. Blanco, A.E. Huespe, R.A. Feijóo, Failure-Oriented Multi-scale Variational Formulation: Micro-structures with nucleation and evolution of softening bands, Comput. Methods Appl. Mech. Eng. 257 (2013) 221-247. https://doi.org/10.1016/j.cma.2012.11.016

[24] S. Toro, P.J. Sánchez, P.J. Blanco, E.A. de Souza Neto, A.E. Huespe, R.A. Feijóo, Multiscale formulation for material failure accounting for cohesive cracks at the macro and micro scales, Int. J. Plast. 76 (2016) 75-110. https://doi.org/10.1016/j.ijplas.2015.07.001

[25] A. Ibrahimbegović, D. Markovič, Strong coupling methods in multi-phase and multi-scale modeling of inelastic behavior of heterogeneous structures, Comput. Methods Appl. Mech. Eng. 192 (2003) 30893107. https://doi.org/10.1016/S0045-7825(03)00342-6

[26] I. Benedetti, M.H. Aliabadi, Multiscale modeling of polycrystalline materials: A boundary element approach to material degradation and fracture, Comput. Methods Appl. Mech. Eng. 289 (2015) 429-453. https://doi.org/10.1016/j.cma.2015.02.018

[27] M. Xiang, J. Chen, Numerical simulation of ductile fracture based on mean field homogenization method: Modeling and implementation, Eng. Fract. Mech. 152 (2016) 147-161. https://doi.org/10.1016/j.engfracmech.2015.07.041

[28] A. Tchalla, W.L. Azoti, Y. Koutsawa, A. Makradi, S. Belouettar, H. Zahrouni, Incremental mean-fields micromechanics scheme for non-linear response of ductile damaged composite materials, Compos. Part B Eng. 69 (2015) 169-180. https://doi.org/10.1016/j.compositesb.2014.08.055

[29] S. Ghosh, J. Bai, D. Paquet, Homogenization-based continuum plasticity-damage model for ductile failure of materials containing heterogeneities, J. Mech. Phys. Solids. 57 (2009) 1017-1044. https://doi.org/10.1016/j.jmps.2009.04.002

[30] D.O. Fernandino, A.P. Cisilino, S. Toro, P.J. Sanchez, Multi-scale analysis of the early damage mechanics of ferritized ductile iron, Int. J. Fract. 207 (2017) 1-26. https://doi.org/10.1007/s10704-0170215-1

[31] V. Le Corre, N. Brusselle-Dupend, M. Moreaud, Numerical modeling of the effective ductile damage of macroporous alumina, Mech. Mater. 114 (2017) 161-171. https://doi.org/10.1016/j.mechmat.2017.08.002

[32] M.G.D. Geers, V.G. Kouznetsova, W.A.M. Brekelmans, Multi-scale computational homogenization: Trends and challenges, J. Comput. Appl. Math. 234 (2010) 2175-2182. https://doi.org/10.1016/j.cam.2009.08.077

[33] K. Matouš, M.G.D. Geers, V.G. Kouznetsova, A. Gillman, A review of predictive nonlinear theories for multiscale modeling of heterogeneous materials, J. Comput. Phys. 330 (2017) 192-220. https://doi.org/10.1016/j.jcp.2016.10.070

[34] S. Hafner, T. Luther, J. Unger, S. Eckardt, C. Konke, Multiscale Simulation Methods in Damage Prediction of Brittle and Ductile Materials, Int. J. Multiscale Comput. Eng. 8 (2010) 17-36. https://doi.org/10.1615/IntJMultCompEng.v8.i1.30

[35] T. Lesičar, J. Sorić, Z. Tonković, Ductile Damage Modelling of Heterogeneous Materials Using a TwoScale Computational Approach, Comput. Methods in Appl. Mech. and Eng. 355 (2019) 113-134. https://doi.org/10.1016/j.cma.2019.06.013

[36] F. Putar, J. Sorić, T. Lesičar, Z. Tonković, A Multiscale Method for Damage Analysis of Quasi-Brittle Heterogeneous Materials, Computer Modelling in Engineering \& Sciences 200 (1) (2019) 123-156. https://doi.org/10.32604/cmes.2019.06562

[37] ABAQUS, ABAQUS/Standard (2014).

[38] R.H.J. Peerlings, Enhanced damage modelling for fracture and fatigue, Ph. D. thesis, University of Technology (1999) Eindhoven, Netherlands.

[39] J. Mazars, G. Pjaudier-Cabot, Continuum damage theory-application to concrete, J. Eng. Mech. 115 (2) (1989) 345-365. https://doi.org/10.1061/(ASCE)0733-9399(1989)115:2(345) 
[40] J.H.P. de Vree, W.A.M. Brekelmans, M.A.J. van Gils, Comparison of nonlocal approaches in continuum damage mechanics, Comp. Struct. 55 (1995) 581-588. https://doi.org/10.1016/0045-7949(94)00501-S

[41] T. Lesičar, Z. Tonković, J. Sorić, A second-order two-scale homogenization procedure using $C^{1}$ macrolevel discretization, Comp. Mech. 54 (2) (2014) 425-441. https://doi.org/10.1007/s00466-0140995-3

[42] T. Lesičar, Multiscale modelling of heterogeneous materials using second-order homogenization, Ph.D. Thesis, Faculty of Mechanical Engineering and Naval Architecture, (2015) Zagreb, Croatia.

[43] Engelen, R. A. B., Geers, M. G. D., Baaijens, F. P. T., Nonlocal implicit gradient-enhanced elastoplasticity for the modelling of softening behaviour, International Journal of Plasticity 19 (4) (2003). 403433. https://doi.org/10.1016/S0749-6419(01)00042-0

[44] T. Lesičar, J. Sorić, Z. Tonković, Large strain, two-scale computational approach using C1 continuity finite element employing a second gradient theory. Computer Methods in Applied Mechanics and Engineering, 298 (2016) 303-324. https://doi.org/10.1016/j.cma.2015.09.017

[45] J. Sorić, T. Lesičar, , Z. Tonković, On Ductile Damage Modelling of Heterogeneous Materials Using Second-order Homogenization Approach, Computer Modelling in Engineering \& Sciences 126 (3) (2021) 915-934. https://doi.org/10.32604/cmes.2021.014142

[46] H.J. Logarzo, G. Capuano, J.J. Rimoli, Smart constitutive laws: Inelastic homogenization through machine learning, Computer Methods in Applied Mechanics and Engineering. 373 (2021) 113482. https://doi.org/10.1016/j.cma.2020.113482

Submitted: $\quad$ 22.11.2021. Jurica Sorić

Tomislav Lesičar

Accepted: $\quad$ 19.12.2021. Zdenko Tonković

Faculty of Mechanical Engineering and Naval Architecture, University of

Zagreb, Ivana

Lučića 5, 10000 Zagreb, Croatia,

e-mail: jurica.soric@fsb.hr, tomislav.lesicar@fsb.hr, zdenko.tonkovic@fsb.hr

Filip Putar

AVL-AST d.o.o.,

Strojarska cesta 22, 10000 Zagreb, Croatia,

e-mail: filip.putar@gmail.com 